\title{
Quantitative Microscopy of Hepatic Changes Induced by Phenethyl Isothiocyanate in Fischer-344 Rats Fed Either a Cereal-Based Diet or a Purified Diet*
}

\author{
Robert H. Gray, ${ }^{1}$ Gabriela Adam-Rodwell, ${ }^{2}$ Dorina Maris, ${ }^{1}$ \\ JEFFREY R. HASKINS, ${ }^{1}$ AND GARY D. STONER ${ }^{2,3}$ \\ ${ }^{1}$ Department of Environmental and Industrial Health, University of Michigan, \\ Ann Arbor, Michigan 48109, and \\ ${ }^{2}$ Department of Pathology, Medical College of Ohio, Toledo, Ohio 43699
}

\begin{abstract}
Hepatic changes induced by phenethyl isothiocyanate (PEITC) in the liver of rats were determined by quantitative microscopy. Groups of male Fischer-344 rats were fed either a standard, cereal-based diet (Wayne rodent meal) or a purified diet (AIN-76A) containing PEITC at concentrations of 0.75 and $6.0 \mathrm{mmol} / \mathrm{kg}$ for $13 \mathrm{wk}$. Severe hepatic lipidosis was observed in control rats fed the purified diet. Addition of PEITC to the purified diet significantly reduced lipid content in hepatocytes. In contrast, lipid content in the liver of the rats fed the cereal-based diet containing PEITC was greater than in control rats maintained on the same diet. In addition, dose-related reductions in hepatocyte, lipid droplet, peroxisome, and mitochondrial volumes were observed in PEITC-treated rats fed the cereal-based diet. These results indicate that PEITC exerts differential effects on the liver of rats fed either the cereal-based or purified diet.
\end{abstract}

Keywords. Morphometric analysis; AIN-76A purified diet; hepatic fatty change; liver; rat

\section{INTRODUCTION}

Phenethyl isothiocyanate (PEITC) occurs naturally as a glucosinolate (gluconasturtiin) in cruciferous and other related plants $(21,22)$. Gluconasturtiin yields PEITC upon hydrolysis catalyzed by thioglucosidase, an enzyme present in these plants and in the intestinal flora of animals (15). Recent studies have demonstrated that PEITC has a marked inhibitory activity toward nitrosamine-induced lung tumorigenesis in rats and mice and esophageal tumorigenesis in rats $(13,14,20)$. Ideally, an inhibitor of chemical carcinogenesis should be effective at nontoxic concentrations. Previously reported data on the potential toxic effects of PEITC are limited $(1,6,14,17)$. An earlier report (1) from this laboratory details the toxicological evaluation of PEITC in Fischer-344 (F-344) rats after single treatment by

\footnotetext{
* Address correspondence to: Dr. Robert H. Gray, Department of Environmental and Industrial Health, The University of Michigan, 3537 SPH-I, Ann Arbor, Michigan 48109-2029.

${ }_{3}$ Present address: The Ohio State University, Department of Preventive Medicine, Arthur James Cancer Hospital and Research Institute, Columbus, Ohio 43210.
}

gavage and continuous treatment in the diet for 13 wk. The results of the dietary subchronic study suggested that the liver may be a target organ for PEITC effects. Any potential toxic effects of PEITC were difficult to assess because the AIN-76A purified diet, used extensively in carcinogenesis and chemoprevention research, caused a marked hepatic lipidosis, predominantly in the periportal region of the liver. The addition of PEITC to the purified diet resulted in a reduction of lipids in the periportal region and a mild to moderate increase of lipid deposition in hepatocytes around the central vein. Morphometric analysis of rat liver from this 13-wk study further demonstrated the attenuated fatty change associated with the presence of PEITC in the purified diet (6).

Based on these results, a morphometric study was conducted to evaluate ultrastructural changes in the liver of rats administered PEITC in the Wayne cereal-based diet or in the AIN-76A purified diet. A random panlobular morphometric sampling scheme was applied to standardize for the differential lobular response previously observed and to optimize the chance of detecting significant compound-related effects. 


\section{Methods AND Materials}

Chemicals and Diets. PEITC (99\% purity, MW 163.24, density 1.094) was purchased from Aldrich Chemical Company (Milwaukee, WI). The AIN-76A purified diet was supplied by Teklad Diets (Madison, WI). Wayne rodent meal (\#8640), the cerealbased diet, was purchased from Wayne Research Animal Care (Chicago, IL).

Animals. F-344 rats were obtained from Harlan Sprague Dawley, Inc. (Indianapolis, IN). The rats were 5-6 wk of age and weighed approximately 100 $\mathrm{g}$ when the study was initiated. Animals were housed 2 per cage in an environmentally controlled room $\left(20^{\circ} \pm 2^{\circ} \mathrm{C} ; 55 \pm 15 \%\right.$ relative humidity, $10-12$ fresh air changes per hour; and a 12-hr light/dark cycle). Rats were acclimated to the laboratory for 7 days prior to initiation of experimental procedures.

Preparation of Test Diets. Specified amounts of PEITC were added to the Wayne meal and the AIN$76 \mathrm{~A}$ diet and mixed in a Hobart mixer to ensure proper homogeneity. Test diets were prepared every 2 wk and stored at $4^{\circ} \mathrm{C}$ until use.

Experimental Design. Groups of 6 male rats were fed either the AIN-76A purified diet or the Wayne rodent meal containing PEITC at a concentration of $0,0.75$, or $6.0 \mathrm{mmol} / \mathrm{kg}$ for $13 \mathrm{wk}$. The dosage levels of PEITC were selected based on a previous 13-wk study of PEITC in F-344 rats $(1,6,14)$. Calculated average daily intake of PEITC was 8 and 67 $\mathrm{mg} / \mathrm{kg}$ body weight in the groups fed the Wayne diet containing 0.75 and $6.0 \mathrm{mmol} / \mathrm{kg}$ PEITC, respectively. Correspondingly, the average daily intake was 7 and $56 \mathrm{mg} / \mathrm{kg}$ body weight of PEITC in the treatment groups fed the AIN-76A diet. Diet and tap water were provided ad libitum throughout the study. The rats were observed daily for overt signs of toxicity. Body weight and food consumption were measured weekly.

Tissue Preparation, Electron Microscopy, and Morphometry. Following 13 wk of treatment, the rats were sacrificed with carbon dioxide and necropsies were performed. Livers were weighed and examined macroscopically. Liver tissue from 4 animals per group was collected and processed for electron microscopic examination and morphometric analysis. A small piece of tissue was removed from the liver of each animal; immersed in a modified Karnovsky's fixative consisting of $2 \%$ glutaraldehyde, $2 \%$ paraformaldehyde in $0.2 \mathrm{M}$ cacodylate buffer at 705 milliosmoles and $\mathrm{pH} 7.3$; minced into $1-\mathrm{mm}^{3}$ blocks; fixed in $1 \%$ osmium tetroxide; dehydrated in a graded ethanol series; and embedded in Spurr's resin. Thick sections $(1 \mu \mathrm{m})$ were cut using a Reichert Om-U2 ultramicrotome with a glass knife and stained with Toluidine blue. Thin sections were cut using a diamond knife, poststained with uranyl acetate and lead citrate, and examined in a Zeiss EM 10CA electron microscope at $80 \mathrm{kV}$.

Four tissue blocks from each animal were selected for the quantitative assessment of liver changes. Cellular parameters were measured by light microscopy at a final magnification of $\times 2,830$ on 4 randomly selected fields per thick section per block. A BioQuant Advanced System IV image analysis system with a calibrated video overlay was utilized to interface with the light microscope. Approximately 8 electron micrographs per block, taken at $\times 4,000$ and enlarged to a final magnification of $\times 20,000$, were used to determine subcellular parameters. Calibration was monitored with a grating replica photographed with each set of negatives. Approximately 384 fields from the thick sections and 750 electron micrographs were used to generate the data presented. The morphometric formulas were based on Weibel et al (23) and adapted for our laboratory computer (7). Differences between groups were determined using one-way analysis of variance (ANO$\mathrm{VA})$ at a significance level of $5 \%$.

\section{RESUlTS}

\section{Hepatocyte Morphology}

Low-magnification electron micrographs of rat liver from each group are shown in Fig. 1. Hepatocytes from rats fed the control cereal-based Wayne diet alone or with either 0.75 or $6.0 \mathrm{mmol} / \mathrm{kg}$ PEITC are shown in Fig. 1A-C, respectively. Hepatocytes from the PEITC treatment groups had a higher lipid droplet content than the control diet group (Fig. 1A). Liver of animals from the $0.75-\mathrm{mmol} / \mathrm{kg}$ PEITC group had more lipid droplets than the $6.0-\mathrm{mmol} /$ $\mathrm{kg}$ PEITC group. Hepatocytes from rats fed the control purified AIN-76A diet are shown in Fig. 1D. The AIN-76A diet produced a marked increase in hepatic lipid accumulation compared to the Wayne diet-fed animals. Liver cells from rats fed the purified AIN-76A diet with $0.75 \mathrm{mmol} / \mathrm{kg}$ PEITC are shown in Fig. 1E. Addition of $0.75 \mathrm{mmol} / \mathrm{kg}$ PEITC reduced the quantity of lipid droplets compared to the control animals in the same group (Fig. 1D). Hepatocytes from rats fed the purified AIN-76A diet with $6.0 \mathrm{mmol} / \mathrm{kg}$ PEITC (Fig. 1F) showed a further reduction of lipid droplets compared to the 0.75 $\mathrm{mmol} / \mathrm{kg}$ PEITC dose level or the AIN-76A control groups. A summary of the hepatic cellular and subcellular morphologic changes is provided in Table $\mathrm{I}$.

\section{Cell and Nuclear Volume Morphometric Parameters}

The mean hepatocyte volume was significantly reduced by 14 and $23 \%$ in rats fed the Wayne diet containing PEITC at concentrations of 0.75 and 6.0 $\mathrm{mmol} / \mathrm{kg}$, respectively (Fig. 2A). Mean hepatocyte 

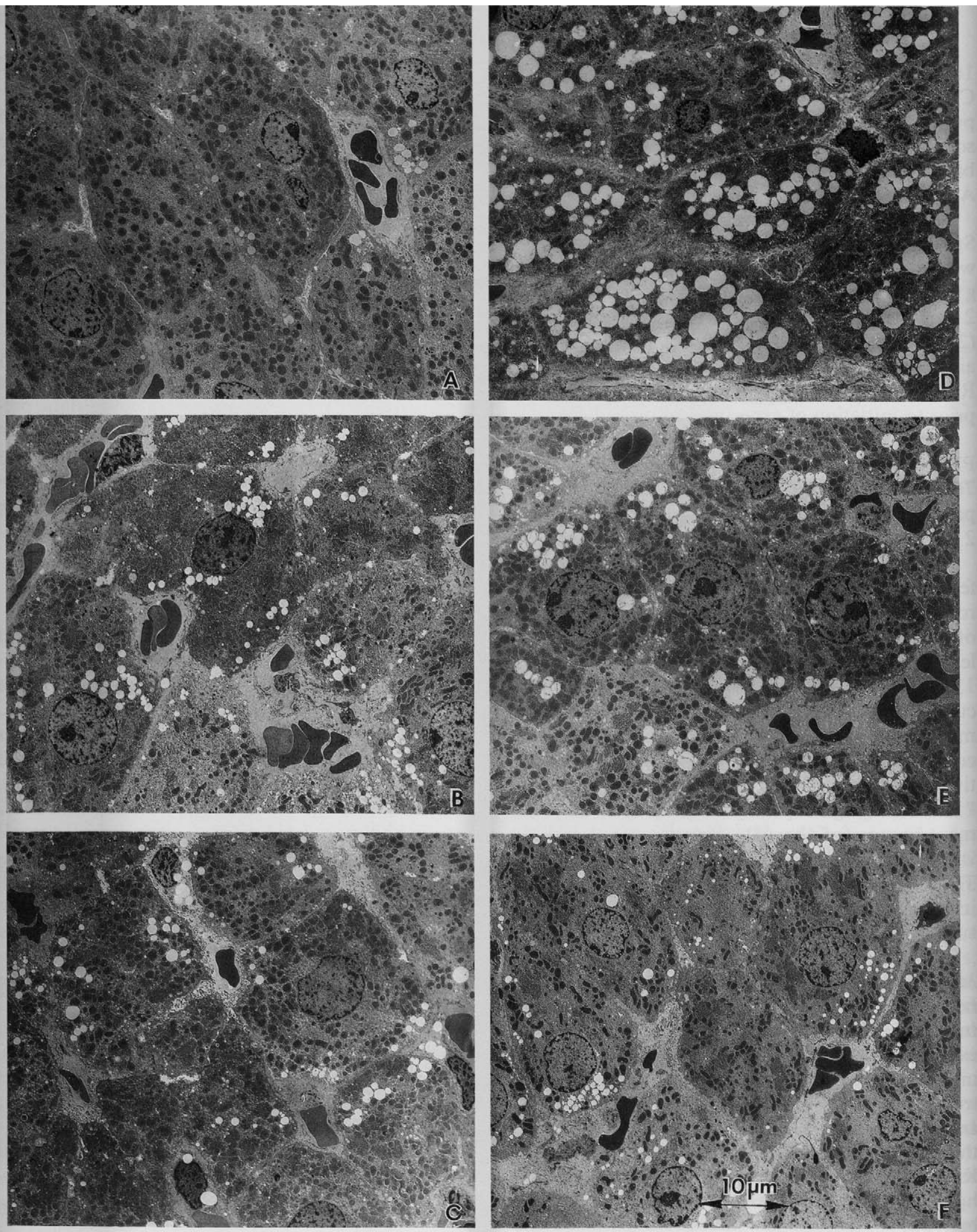

FIG. 1.-Representative electron micrographs of all treatment groups. $\times 1,600$. A) Hepatocytes from rats fed the cerealbased Wayne diet. B) Hepatocytes from rats fed the Wayne diet with $0.75 \mathrm{mmol} / \mathrm{kg}$ PEITC. C) Hepatocytes from rats fed the Wayne diet with $6.0 \mathrm{mmol} / \mathrm{kg}$ PEITC. D) Hepatocytes from rats fed the purified AIN-76A diet. E) Hepatocytes from rats fed the purified AIN-76A diet with $0.75 \mathrm{mmol} / \mathrm{kg}$ PEITC. F) Hepatocytes from rats fed the purified AIN-76A diet with $6.0 \mathrm{mmol} / \mathrm{kg}$ PEITC. 

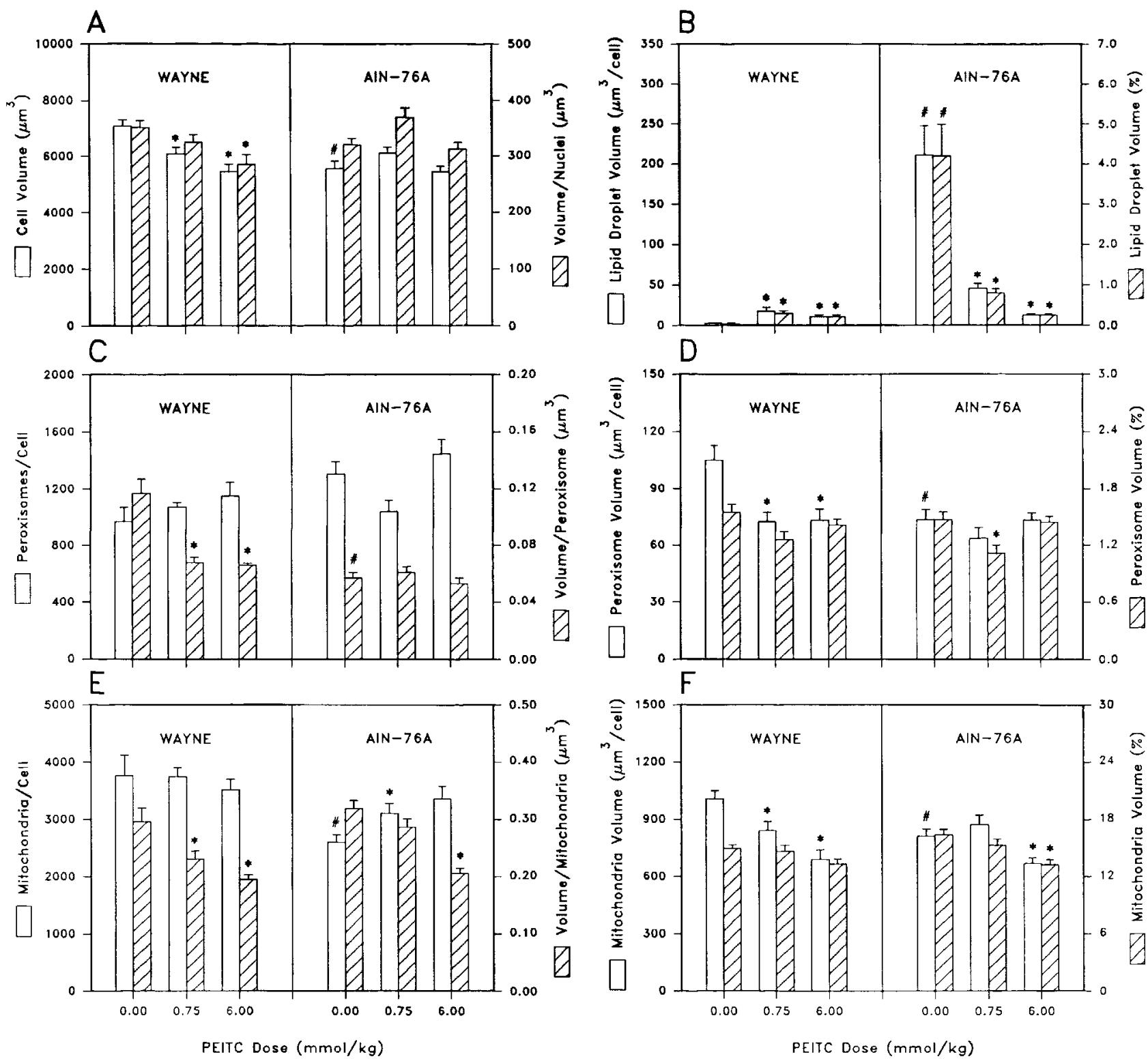

FIG. 2. - Morphometric hepatic changes induced by PEITC in F-344 rats. A) Comparison of the effects of PEITC treatment on mean cell volume $\left(\mu \mathrm{m}^{3}\right)$ and mean nuclear volume $\left(\mu \mathrm{m}^{3}\right)$ in liver of rats fed either the Wayne or AIN-76A purified diet. B) Comparison of the effects of PEITC treatment on lipid droplet volume $\left(\mu \mathrm{m}^{3}\right)$ per cell and lipid volume fraction (\%) per cell in the liver of rats fed PEITC in the Wayne diet or in the AIN-76A purified diet. C) Comparison of the effects of PEITC treatment on peroxisome number per cell and the mean volume per peroxisome $\left(\mu \mathrm{m}^{3}\right)$ in hepatocytes of rats administered PEITC in the Wayne diet or in the AIN-76A purified diet. D) Comparison of the effects of PEITC treatment on peroxisome volume per cell $\left(\mu \mathrm{m}^{3}\right)$ and the volume fraction percentage per cell in hepatocytes of rats administered PEITC in the Wayne diet or in the AIN-76A purified diet. E) Comparison of the effects of PEITC treatment on the mean number of mitochondria per cell and the mean volume per mitochondria $\left(\mu \mathrm{m}^{3}\right)$ in hepatocytes of rats exposed to PEITC in the Wayne diet or in the AIN-76A purified diet. F) Comparison of the effects of PEITC treatment on the mean mitochondria volume $\left(\mu \mathrm{m}^{3}\right)$ per cell and the mean mitochondria volume fraction percentage per cell in hepatocytes of rats fed PEITC in the Wayne diet or the AIN-76A purified diet. Error based on histograms represent \pm standard errors of the mean for each parameter measured. *Statistically significant versus the corresponding control group ( $p<0.05$; one-way ANOVA); "Statistically significant versus the Wayne control diet group $(p<0.05$; one-way ANOVA). 
volume in the Wayne diet control animals was 7,085 $\mu \mathrm{m}^{3}$ compared to 6,101 and $5454 \mu \mathrm{m}^{3}$, respectively, in the low- and high-dose PEITC-treated animals. Hepatocyte volume in control rats fed AIN-76A diet was $5,561 \mu \mathrm{m}^{3}$, a significant reduction when compared to $7,085 \mu \mathrm{m}^{3}$ observed in control rats fed the Wayne diet. The AIN-76A-fed rats treated with PEITC did not show significant differences in mean hepatocyte volume from their respective controls.

The mean nuclear volume decreased by 8 and $19 \%$ in rats fed the Wayne diet containing PEITC at 0.75 and $6.0 \mathrm{mmol} / \mathrm{kg}$, respectively; the mean nuclear volume in the high-dose PEITC group, 285 $\mu \mathrm{m}^{3}$, was significantly reduced when compared to controls, $352 \mu \mathrm{m}^{3}$ (Fig. 2A). Addition of PEITC to the AIN-76A diet did not induce significant changes in the cell or nuclear volume (Fig. 2A). The AIN$76 \mathrm{~A}$-fed group treated with PEITC did not show significant differences from the control group for nuclear volume.

\section{Lipid Droplet Morphometric Parameters}

Figure 2B summarizes the results of morphometric lipid droplet parameters. Significant differences in lipid droplet content were observed between the control Wayne and control AIN-76A diet groups as well as within groups administered PEITC. The mean lipid droplet volume per cell in rats fed the Wayne diet was the lowest of any of the groups, that is, $2.8 \mu \mathrm{m}^{3}$ per cell or $0.04 \%$ of the total cell volume. The addition of PEITC to the Wayne diet resulted in differential effects depending on the dose. At $0.75 \mathrm{mmol} / \mathrm{kg}$ PEITC, the mean lipid droplet volume per cell increased to $17.7 \mu \mathrm{m}^{3}$, or $0.30 \%$ of the total cell volume. In contrast, at $6.0 \mathrm{mmol} / \mathrm{kg}$ PEITC, the mean lipid droplet volume per cell decreased to $10.9 \mu \mathrm{m}^{3}$, or $0.22 \%$ of the total cell volume. The mean lipid droplet volume per hepatocyte of rats fed the AIN-76A diet was more than 70-fold greater than that of rats fed the Wayne diet (Figs. $1 \mathrm{~A}, \mathrm{D}$, and 2B). The mean lipid droplet volume per cell increased from 2.8 to $211 \mu \mathrm{m}^{3}$, and the mean lipid droplet volume fraction increased from 0.4 to $4.2 \%$ in rats fed the AIN-76A purified diet.

PEITC in the AIN-76A diet caused an inverse dose response for lipid droplet formation (Fig. 2B). The mean lipid droplet volume per cell was reduced from $211 \mu \mathrm{m}^{3}$ in control animals to $45.5 \mu \mathrm{m}^{3}$ in rats treated with $0.75 \mathrm{mmol} / \mathrm{kg}$ PEITC; these changes correspond to a reduction in mean lipid droplet volume per cell from $4.20 \%$ in controls to $0.80 \%$ in the low-dose group. At $6.0 \mathrm{mmol} / \mathrm{kg}$ PEITC, the mean hepatocyte lipid droplet volume was further reduced to $12.7 \mu \mathrm{m}^{3}$, corresponding to a $0.25 \%$ lipid droplet volume per cell.

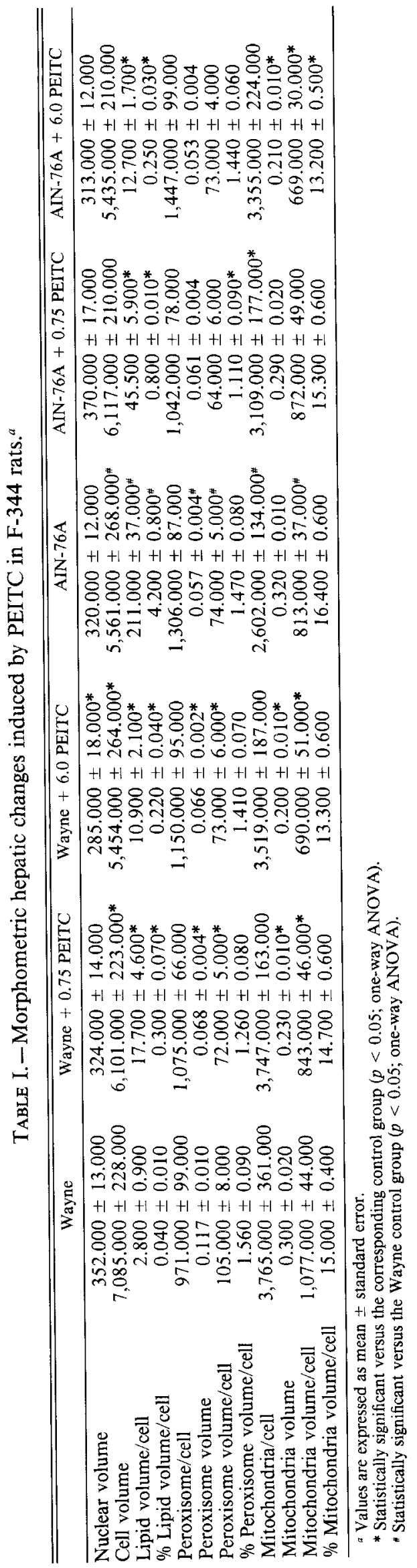




\section{Peroxisome Morphometric Parameters}

Peroxisome morphometric parameters are shown in Fig. 2C and D. In rats fed the Wayne diet, the mean peroxisome number per cell was not significantly different after PEITC treatment. However, the mean number of peroxisomes per cell showed slight dose-related increases from 971 in controls to 1,075 and 1,150 in the $0.75-$ and $6.0-\mathrm{mmol} / \mathrm{kg}$ PEITC dose groups, respectively (Fig. 2C). Mean peroxisome volume significantly decreased in the PEITC-treated groups. Mean peroxisome volume was $0.117 \mu \mathrm{m}^{3}$ in control animals and was reduced by 42 and $45 \%$ to 0.068 and $0.066 \mu \mathrm{m}^{3}$ in the lowand high-dose PEITC groups, respectively (Fig. 2C).

In rats fed the AIN-76A diet, the mean number of peroxisomes per cell was greater than in Wayne diet animals, 1,306 compared to 971 , respectively. AIN-76A-fed rats treated with PEITC showed a slight, but not statistically significant, change in the mean number of peroxisomes per cell, from 1,306 in control to 1,042 and 1,447 in the 0.75 - and 6.0$\mathrm{mmol} / \mathrm{kg}$ PEITC dose levels, respectively (Fig. 2C).

In the Wayne-fed groups, the mean volume per peroxisome was reduced from $0.117 \mu \mathrm{m}^{3}$ in controls to 0.068 and $0.066 \mu \mathrm{m}^{3}$ in the $0.75-$ and $6.0-\mathrm{mmol} /$ $\mathrm{kg}$ PEITC dose groups, respectively. The mean volume per peroxisome of the AIN-76A group did not change with PEITC treatment at either dose (Fig. 2C). The mean volume per peroxisome in control animals of the AIN-76A diet was $0.057 \mu \mathrm{m}^{3}$ compared to $0.117 \mu \mathrm{m}^{3}$ in the Wayne-fed control rats (Fig. 2C). The mean peroxisome volume per cell in animals fed the Wayne diet showed a reduction from $105 \mu \mathrm{m}^{3}$ in control rats to 72 and $73 \mu \mathrm{m}^{3}$, respectively, in the $0.75-$ and $6.0-\mathrm{mmol} / \mathrm{kg}$ PEITC treatment groups (Fig. 2D). In the AIN-76A-fed control rats, the mean peroxisome volume per cell was 74 $\mu \mathrm{m}^{3}$, a significant reduction from the Wayne-fed animals, $105 \mu \mathrm{m}^{3}$. The mean peroxisome volume per cell was not significantly different in the rats fed the AIN-76A diet containing PEITC.

The mean peroxisome volume fraction per cell of rats fed the control Wayne diet was $1.56 \%$ (Fig. 2D). The peroxisome volume fraction was not changed by the addition of PEITC to the diet. In the AIN$76 \mathrm{~A}-\mathrm{fed}$ rats, the $0.75-\mathrm{mmol} / \mathrm{kg}$ PEITC treatment produced a significant decrease in mean peroxisome volume fraction per cell to $1.11 \%$ compared to $1.47 \%$ in the control group (Fig. 2D). The mean peroxisome volume percent fractions per cell of the Wayne and AIN-76A control groups were not statistically different.

\section{Mitochondria Morphometric Parameters}

The mitochondria morphometric parameters are shown in Fig. 2E and F. The mean number of $\mathrm{mi}-$ tochondria per hepatocyte in control rats fed Wayne diet was 3,765 (Fig. 2E). Rats fed the Wayne diet containing PEITC showed no significant change in the number of mitochondria per cell. In the Waynefed groups, the mean volume per mitochondria was significantly decreased from $0.30 \mu \mathrm{m}^{3}$ in controls to 0.23 and $0.20 \mu \mathrm{m}^{3}$ in the $0.75-$ and $6.0-\mathrm{mmol} / \mathrm{kg}$ PEITC treatment groups (Fig 2E).

Among the AIN-76A-fed rats, the mean number of mitochondria per hepatocyte was increased in both PEITC treatment groups; however, only the $0.75-\mathrm{mmol} / \mathrm{kg}$ PEITC group showed a significant increase, that is, 3,109 compared to 2,602 in the control group (Fig. 2E). The mean number of $\mathrm{mi}-$ tochondria per cell in the AIN-76A control group was significantly lower than in the Wayne control group, that is, 2,602 compared to 3,765 , respectively.

The mean volume per mitochondria in rats fed AIN-76A diet decreased from $0.32 \mu \mathrm{m}^{3}$ in the controls to $0.21 \mu \mathrm{m}^{3}$ in the $6-\mathrm{mmol} / \mathrm{kg}$ PEITC (Fig 2F). No significant differences were observed in the mean mitochondria volume between the control groups of the Wayne- and AIN-76A-fed animals.

In rats fed the Wayne diet, PEITC treatment caused a dose-dependent decrease in the mean mitochondria volume per cell (Fig. 2F). The mean mitochondria volume per cell in the control group was $1,007 \mu \mathrm{m}^{3}$ compared to 843 and $690 \mu \mathrm{m}^{3}$ in the $0.75-$ and $6.0-\mathrm{mmol} / \mathrm{kg}$ PEITC treated groups, respectively (Fig 2F). In rats fed the AIN-76A diet, only the $6.0-\mathrm{mmol} / \mathrm{kg}$ PEITC dose group showed a decrease in the mean mitochondrial volume per cell, $669 \mu \mathrm{m}^{3}$, compared to $813 \mu \mathrm{m}^{3}$ per cell in the control group. The mean mitochondria volume per cell in the AIN-76A group, $813 \mu \mathrm{m}^{3}$, was significantly lower than in the Wayne-fed group, 1,007 $\mu \mathrm{m}^{3}$.

The mean mitochondria volume fraction per cell was not significantly changed in any of the Wayne diet groups (Fig. 2F). The mitochondria volume fractions were $15.0,14.7$, and $13.3 \%$ for the control and the $0.75-$ and $6.0-\mathrm{mmol} / \mathrm{kg}$ PEITC dose groups, respectively. In the AIN-76A groups, only the high PEITC dose group showed a significant decrease in the mean mitochondria volume fraction, $13.0 \%$ compared to $16.4 \%$ observed in the control group (Fig. 2F). Comparison of the mean mitochondria volume fraction per cell for the Wayne and AIN$76 \mathrm{~A}$ diet control groups revealed no significant differences for this parameter.

\section{Discussion}

A major observation in this study was severe fatty change in the liver of control rats fed the AIN-76A diet compared to the cereal-based Wayne diet. The percentage of lipid droplet volume per cell was more 
than 100 -fold greater in rats fed the purified diet than in control rats fed the Wayne diet. These results were consistent with previous reports on periportal lipidosis in livers of rats fed the AIN-76A diet (8, 12).

AIN-76A purified diet, formulated by the American Institute for Nutrition (3), is widely used in carcinogenesis and chemoprevention studies. This purified diet contains $50 \%$ sucrose and $15 \%$ starch and is unsuitable for rodent studies in its present formulation. Apparently, the excess sucrose is metabolized to lipids, leading to an imbalance between synthesis and secretion of lipoproteins from hepatocytes into circulating blood. Hamm et al (8) demonstrated that substitution of starch for sucrose eliminated hepatic lipidosis in both F-344 and CD rats. Addition of PEITC to the AIN-76A diet resulted in a substantial reduction of the lipid content in hepatocytes. Other ultrastructural changes induced by PEITC in the liver of rats fed AIN-76A diet included an increased number of mitochondria per cell associated with a decreased mean absolute mitochondria volume per cell.

Ultrastructural changes induced by PEITC in the liver of rats fed the Wayne diet contrasted with those seen in rats fed the AIN-76A diet. Morphometric analysis revealed a dose-dependent trend for reduced hepatocyte volume in rats fed the Wayne diet containing PEITC at both low and high concentrations. No significant effects on liver weight were observed. We also found that the mean lipid droplet volume per hepatocyte and the percentage of lipid droplet volume per hepatocyte was significantly increased in the PEITC-treated groups.

The underlying mechanisms responsible for PEITC-induced changes in the liver are not known. Fatty change is a common lesion in the liver and is morphological evidence of excess accumulation of fat, which appears as intracytoplasmic vacuolation. Lipids reach the liver mainly as alimentary chylomicrons or fatty acids from adipose tissue and are utilized within the liver or are secreted as very low density lipoproteins. There is evidence that fat accumulates in the liver when there is an imbalance among supply, utilization, and secretion of lipids (18). Different chemicals produce fatty change by different means $(2,9,11,18)$. For example, carbon tetrachloride $\left(\mathrm{CCl}_{4}\right)$ and phosphorus impair protein synthesis, resulting in reduced apoproteins available for very low density lipoprotein formation and secretion of triglycerides by the liver $(4,16)$. In choline deficiency, phospholipid metabolism is impaired, resulting in reduced secretion of lipids into the plasma (16). In the current study, it appears that after hepatic lipids are accumulating (Fig. 2B) above baseline of the Wayne-fed controls the higher dose levels of PEITC result in lipid reduction. At the 6.0$\mathrm{mmol} / \mathrm{kg}$ PEITC dose level for both diet groups, lipid levels are reduced compared to the $0.75-\mathrm{mmol} /$ $\mathrm{kg}$ PEITC dose. The exact mechanism of lipid reduction or the possible role of PEITC in this reduction is not evident from data collected and analyzed in these experiments. The changes could be due to inhibition of lipid synthesis or an induction of lipid mobility to reduce the lipid content. In previous studies (1), decreases in serum triglycerides observed following administration of PEITC for 7 or $13 \mathrm{wk}$ supports the possible involvement of PEITC in intracellular lipid reduction.

The mechanisms by which PEITC alters hepatocyte morphology and physiology are not known. It has been shown that isothiocyanates are reactive electrophiles, capable of covalent binding to membrane proteins at critical sites where transmembrane functions may occur $(5,20)$. Investigations on cytological effects of allyl and benzyl isothiocyanate in isolated hepatocytes demonstrated that thiol groups in the plasma membrane and other cellular membrane systems are the primary target for these compounds (20). Cytomorphological changes observed in these experiments consisted of swelling and surface blebbing of mitochondria and dilation of the endoplasmic reticulum. Alteration of the membrane proteins appeared to affect the monovalent cation and water balance of the cell organelles rather than divalent calcium homeostasis. It is possible that PEITC binds to membrane proteins, resulting in reduced protein synthesis, altered lipid metabolism, and excretory and secretory functions of hepatocytes. Reduced hepatocyte and organelle volumes seen in rats fed the Wayne diet containing PEITC may indicate a reduced metabolic rate in which turnover of cellular components continues whereas de novo synthesis decreases.

The accumulation of lipids in hepatocytes of rats fed the Wayne diet containing PEITC suggests an impaired hepatic secretory function, possibly due to a reduced level of lipoproteins. This supposition is supported by the results of 7- and 13-wk dietary toxicity studies in which rats fed PEITC in a cerealbased diet had reduced plasma triglyceride levels at dose levels of $3.0 \mathrm{mmol} / \mathrm{kg}$ and higher (1). Lombardi and Recknagel (10) reported that an alteration in triglyceride secretion from the hepatocyte into the blood is a common factor in toxic liver injury. Similarly, diminished hepatic lipidosis observed in rats administered PEITC in AIN-76A purified diet could be a result of reduced synthesis of triglycerides as a consequence of reduced metabolic rate. It is noteworthy to remark that triglyceride levels in plasma were reduced in rats fed PEITC in AIN-76A for 13 wk (1). 
As indicated in the Introduction of this paper, we conducted a 13-wk study to assess the potential toxicity of PEITC in F-344 rats (1) where the PEITC was administered in AIN-76A diet. Because the diet itself caused severe hepatic lipidosis, it was not possible to detect any toxic effects in the liver that may have been caused by the PEITC. Therefore, we recently conducted a subsequent 13 -wk toxicity study in which PEITC was administered to F-344 rats in NIH-07 diet (personal communication). The results of this study showed that the NIH-07 diet itself produced no observable liver toxicity. Moreover, PEITC produced no observable hepatotoxicity when administered in doses ranging from 3 to $15 \mathrm{mmol} /$ $\mathrm{kg}$ diet [i.e., up to 5 times the dose level used in Adam-Rodwell et al (1)]. Thus, under experimental conditions where any potential hepatotoxicity could be ascertained, PEITC produced no apparent toxicity. These results suggest that, with respect to the liver, PEITC can be tolerated at dose levels that far exceed doses that exhibit effective chemopreventive activity.

\section{ACKNOWLEDGMENTS}

The authors thank Dr. William Gunning for his helpful comments and Mr. Edward Calomeni, Mr. Bradley Nelson and Ms. Kimberly Brosamer for their technical assistance. This study was supported by National Cancer Institute grant CA46535.

\section{REFERENCES}

1. Adam-Rodwell G, Conran PB, Mandal S, and Stoner GD (1990). 13-Week toxicity study of phenyl and phenethyl isothiocyanates in Fischer 344 rats fed AIN76A diet. Toxicologist 10: 300.

2. Andrews LS and Snyder R (1986). Toxic effects of solvents and vapors. In: Casarett and Doull's Toxicology, 3rd ed., CD Klaassen, MO Amdur, and Doull J (eds). Macmillan, New York, pp. 645-648.

3. Bieri JG, Stoews GS, Briggs GM, Phillips RW, Woodard JC, and Knapka JJ (1977). Report of the American Institute of Nutrition Ad Hoc Committee on Standards for Nutritional Studies. J. Nutr. 107: 1340 1348.

4. Bratin WJ, Glende EA Jr, and Recknagel RO (1985). Pathological mechanisms in carbon tetrachloride hepatotoxicity. Free Radicals Biol. Med. 1: 27-38.

5. Bruggeman IM, Temmink JHM, and van Bladeren PJ (1986). Glutathione- and cysteine-mediated cytotoxicity of allyl and benzyl isothiocyanate. Toxicol. Appl. Pharm. 83: 349-359.

6. Gray RH, Crowfoot SD, Randall J, Haskins JR, Adam-Rodwell G, and Stoner GD (1990). Hepatic ultrastructural changes induced by phenyl and phenethyl isothiocyanates in rats fed AIN-76A diet. Toxicologist 10: 300 .

7. Gray RH and de la Iglesia FA (1984). Quantitative microscopy comparison of peroxisome proliferation by the lipid-regulating agent gemfibrozil in several species. Hepatology 4: 520-530.

8. Hamm TE Jr, Raynor T, and Caviston T (1982). Unsuitability of the AIN 76A diet for male F-344 and $C D$ rats and improvement by substituting starch for sucrose. Lab. Anim. Sci. 32: 414.

9. Kyle ME and Farber JL (1991). Biochemical mechanisms of toxic cell injury. In: Handbook of Toxicologic Pathology, WM Haschek and CG Rousseaux (eds). Academic Press, New York. pp. 71-89.

10. Lombardi B and Recknagel RO (1962). Interference with secretion of triglycerides by the liver as a common factor in toxic liver injury. Am. J. Pathol. 40: 571-586.

11. McGuire EJ, Lucas JA, Gray RH, and de la Iglesia FA (1991). Peroxisome induction potential and lipidregulating activity in rats. Am. J. Pathol. 139: 217229.

12. Medinsky MA, Popp JA, Hamm TE, and Dent JG (1982). Development of hepatic lesions in male Fisher-344 rats fed AIN-76A purified diet. Toxicol. Appl. Pharm. 62: 111-120.

13. Morse MA, Amin SG, Hecht SS, and Chung FL (1989). Effects of aromatic isothiocyanates on tumorigenicity, $\mathrm{O}^{8}$-methylguanine formation, and metabolism of the tobacco-specific nitrosamine, 4-(methylnitrosamino)-1-(3-pyridyl)-1-butanone in $\mathrm{A} / \mathrm{J}$ mouse lung. Cancer Res. 49: 2894-2897.

14. Morse MA, Wang CX, Stoner GD, Mandal S, Conran PB, Amin SG, Hecht SS, and Chung FL (1989). Inhibition of 4-(methylnitrosamino)-1-(3-pyridyl)-1butanone-induced DNA adduct formation and tumorigenicity in the lung of F-344 rats by dietary phenethyl isothiocyanate. Cancer Res. 49: 549-553.

15. Oginsky EL, Stein AE, and Greer MA (1965). Myrosinase activity in bacteria as demonstrated by conversion of progoitrin to goitrin. Proc. Soc. Exp. Biol. Med. 119: 360-364.

16. Slater TF (1982). Activation of carbon tetrachloride: Chemical principles and biological significance. In: Free Radicals, Lipid Peroxidation and Cancer, DCH McBrien and TF Slater (eds). Academic Press, New York, pp. 243-274.

17. Speijers GJA, Danse LHJC, Van Leeuwen FXR, and Loeber JG (1985). Four-week toxicity study of phenyl isothiocyanate in rats. Fd. Chem. Toxic. 23: $1015-$ 1017.

18. Stein O and Stein Y (1967). Lipid synthesis, intracellular transport, storage, and secretion. I. Electron microscopic radioautographic study of liver after injection of tritiated palmitate or glycerol in fasted and ethanol-treated rats. J. Cell Biol. 33: 319-339.

19. Stoner GD, Morrissey DT, Heur YH, Daniel EM, Galati AJ, and Wagner SA (1991). Inhibitory effects of phenethyl isothiocyanate on $\mathrm{N}$-nitrosobenzylmethylamine carcinogenesis in the rat esophagus. Cancer Res. 51: 2063-2068.

20. Temmink JHM, Bruggeman IM. and van Bladeren PJ (1986). Cytomorphological changes in liver cells exposed to allyl and benzyl isothiocyanate and their 
cysteine and glutathione conjugates. Arch. Toxicol. 59: 103-110.

21. Tookey HL, VanEtten $\mathrm{CH}$, and Daxenbichler $\mathrm{ME}$ (1980). Glucosinolates. In: Toxic Constituents of Plant Foodstuffs, IE Liener (ed). Academic Press, New York, pp. 103-142.

22. VanEtten $\mathrm{CH}$, Daxenbichler $\mathrm{ME}$, Williams $\mathrm{PH}$, and Kwolek, WF (1976). Glucosinolates and derived products in cruciferous vegetables. Analysis of the edible part from twenty-two varieties of cabbage. $J$. Agric. Food. Chem. 24: 452-455.

23. Weibel ER, Staubi W, Gnagi HR, and Hess FA (1969). Correlated morphometric and biochemical studies on the liver cell: I. Morphometric model, stereologic methods, and normal morphometric data for rat liver. J. Cell Biol. 42: 68-91. 